\title{
COMPACT MEASURES AND MEASURABLE WEAK SECTIONS
}

\author{
WERNER RINKEWITZ
}

\begin{abstract}
Compact measures, i.e. measures that are inner-regular with respect to a compact family of sets, are related to measurable weak sections in the same way as semicompact measures are related to disintegration. This enables us to prove several stability properties of the class of compact measures. E.g., a countable sum of compact measures is compact; the image $v$ of a compact measure $\mu$ is compact provided $\mu$ is an extremal preimage measure of $v$. As a consequence, we show that every tight Baire measure is compact.
\end{abstract}

\section{Introduction}

Let $(X, \mathscr{A})$ be a measurable space, $(Y, \mathscr{B}, v)$ be a finite measure space, and $p: X \longrightarrow Y$ be an $(\mathscr{A}, \mathscr{B})$-measurable map. If $\mu$ is a measure on $\mathscr{A}$ with $\mu\left(p^{-1}[B]\right)=v(B)$ for all $B \in \mathscr{B}$, we write $p(\mu)=\nu$, and call $\mu$ a preimage measure of $v$; let us denote the convex set of all such measures by $M(p, v)$. One way to solve the equation $p(\mu)=v$ (for $\mu$ ) consists in finding a measurable section $s$ for $p$ (i.e. a map $s: Y \longrightarrow X$ with $p \circ s=\operatorname{id}_{Y}$ ) and then putting $\mu:=s(v)$. It is well-known that in this case we get even an extremal preimage measure of $v$, i.e., $\mu \in \operatorname{ex} M(p, v)$; and this last fact remains true if we replace $s$ by a so-called measurable weak section $q$ for $p$, a notion introduced in [3] (see $\$ 2$ for the definition). Since in general not every $\mu \in \operatorname{ex} M(p, v)$ can be representated in this way ( $\$ 2$, Example 2.1), it is natural to ask in which situation this is true. E.g., S. Graf gives a positive answer for the case that $\mu$ is a Radon measure on a Hausdorff space $X$ ([4, Theorem 3]). In this paper, we show first (Theorem 2.2) that the result of S. Graf holds for a more general class of measures: the compact measures in the sense of [14] (see $\$ 1$ for a definition; cf. also [7, Remark 3]). More important, however, is that we can also prove a converse of this result: If we obtain a given measure $\mu$ for all $p$ and $v$ satisfying $\mu \in \operatorname{ex} M(p, v)$ as the image of $v$ under a measurable weak section for $p$, then $\mu$ is compact (Theorem 2.4).

These two results show that the class of compact measures is related to the notion of a measurable weak section in the same way as the class of semi-

Received May 26, 1999. 
compact measures - i.e. the compact measures in the sense of [9]; here we use the terminology suggested in $[10,1.4$.(d)], cf. also [11] and $\S 1-$ is related to a kind of disintegration introduced by J. Pachl (cf. [10, Theorem 3.5, Theorem 2.2]). As in [10], [11], this connection enables a further investigation of the class of compact measures. E.g., we can show that a countable sum of compact measures is compact again. While in this aspect the behaviour of compact measures is analogous to that of the semicompact ones, the same is not true for another important operation: The image $v=p(\mu)$ of a compact measure $\mu$ is in general not compact ( $\$ 2$, Example 2.5). But, if in addition $\mu \in \operatorname{ex} M(p, v)$, then $v$ is compact (Theorem 2.6). By this invariance property, we prove a result that was implicitly shown in [1] (Proposition 3.1) for completely regular Hausdorff spaces: Every tight Baire measure is compact (cf. Corollary 2.8 and Remark 1.2(a)). Hence compact measures are a common generalization of finite Radon measures (in the sense of [15, p. 82]) and tight Baire measures on arbitrary topological spaces.

Of course, the significance of these considerations stems finally from the fact that some theorems in measure theory hold for compact measures in general: Let us mention, e.g., the results in [7] (Theorems 1, 2, 3) on measurable selections and uncountable measurable unions, the representation of compact measures by Loeb measures ([14, Theorem 1.1], [13, Corollary 4.5]), or our Theorem 2.2. In this sense, the present work is addressed to the question: Which measures are compact?

\section{Preliminaries and first results on compact measures}

Before we introduce the main subject of this paper, the class of compact measures, let us fix some general notations. Let $A$ be a subset of some set $X$, and let $\mathscr{B}$ be a collection of subsets of $X$. We write $A^{c}$ for the complement of $A$ in $X$ and $\chi_{A}$ for the characteristic function of $A ; \mathscr{B}^{c}:=\left\{B^{c} \mid B \in \mathscr{B}\right\}, \mathscr{B}_{\delta}$ denotes the class of all countable intersections of members of $\mathscr{B}$, and $\sigma(\mathscr{B})$ is the $\sigma$-algebra generated by $\mathscr{B}$. If $\mathscr{B}$ contains $\emptyset, X$ and is closed under finite unions and finite (countable) intersections, we call $\mathscr{B}$ a $(\delta$-)lattice. $\mathscr{B}$ has the finite intersection property (f.i.p.) provided every finite subset of $\mathscr{B}$ has nonempty intersection. $\mathscr{B}$ is called compact (semicompact) if every (countable) $\mathscr{B}_{0} \subset \mathscr{B}$ with f.i.p. has nonempty intersection. Let $(X, \mathscr{A}, \mu)$ be a measure space; throughout our paper this means that $\mu$ is nonnegative and finite. The completion of $\mathscr{A}$ with respect to $\mu$ is denoted by $\mathscr{A}_{\mu}$; whenever necessary, we will identify $\mu$ with its completion on $\mathscr{A}_{\mu}$. We write $A \subset_{\mu} B$ provided $\mu(A \backslash B)=0$. Similarly, we define $A={ }_{\mu} B$ and $f={ }_{\mu} g$ for $\mathscr{A}_{\mu}$-measurable functions $f, g: X \longrightarrow$ R. The measure $\mu$ is called $\mathscr{K}$-regular or regular with respect to $\mathscr{K}$ (where $\mathscr{K} \subset \mathscr{A}$ ) provided $\mu(A)=\sup \{\mu(K) \mid K \in \mathscr{K}, K \subset A\}$ for all $A \in \mathscr{A}$; the measure 
space $(X, \mathscr{A}, \mu)$ or the measure $\mu$ is compact (semicompact) if there exists a compact (semicompact) lattice $\mathscr{K} \subset \mathscr{A}$ such that $\mu$ is $\mathscr{K}$-regular. (By [14, Corollary 3.2], our definition is equivalent to that given in [14] or [7]; however, it is often convenient to assume that $\mathscr{K}$ is a lattice.)

Basic examples of compact measures are finite Radon measures, i.e. measures defined on the Borel $\sigma$-algebra of a topological space $X$ that are regular with respect to the family of closed compact sets in $X$, and, of course, the Dirac measures.

The main tool of our investigation is Proposition 1.1. For this it is necessary to introduce, similar to $[10, \S 2]$, the Stone representation space $S(\mathscr{A})$ of a $\sigma$-algebra $\mathscr{A}$. We summarize some well-known facts concerning it: $S(\mathscr{A})$ is a compact zero dimensional Hausdorff space, which is, up to a homeomorphism, uniquely determined by the condition that the algebra of its clopen sets, $\mathscr{D}(S(\mathscr{A}))$, is isomorphic to $\mathscr{A}$. Let in the sequal

$$
\widehat{\imath} \mathscr{A} \ni A \mapsto \widehat{A} \in \widehat{\mathscr{A}}:=\mathfrak{D}(S(\mathscr{A}))
$$

be such an algebra isomorphism. Furthermore, with every measure $\mu$ on $\mathscr{A}$ we can associate a measure $\widehat{\mu}$ on $\sigma(\widehat{\mathscr{A}})$ in a natural way: Put $\widehat{\mu}(\widehat{A}):=\mu(A)$ for all $A \in \mathscr{A}$; then $\widehat{\mu}$ is additive on $\widehat{\mathscr{A}}$ and $\sigma$-smooth at $\emptyset$. Therefore $\widehat{\mu}$ can uniquely be extended to a measure $\widehat{\mu}$ on $\sigma(\widehat{A})$.

Proposition 1.1. Let $(X, \mathscr{A}, \mu)$ be a measure space, and let $\mathscr{L} \subset \mathscr{A}$ be a $\delta$-lattice such that $\mu$ is $\mathscr{L}$-regular. The following conditions are equivalent:

(i) $\mu$ is compact.

(ii) For every measure space $(Y, \mathscr{B}, v)$ and every algebra homomorphism $\Phi: \mathscr{A} \longrightarrow \mathscr{B}_{\nu}$ satisfying $v \circ \Phi=\mu$, there exists a $\left(\mathscr{B}_{\nu}, \mathscr{A}\right)$-measurable map $q: Y \longrightarrow X$ such that $\Phi(A)={ }_{v} q^{-1}[A]$ for all $A \in \mathscr{A}$.

(iii) There exists a $\left(\sigma(\widehat{\mathscr{A}})_{\hat{\mu}}, \mathscr{A}\right)$-measurable map $q: \widehat{X} \longrightarrow X$ such that $\widehat{A} \subset \hat{\mu} q^{-1}[A]$ for all $A \in \mathscr{A}$.

(iv) There exists a compact lattice $\mathscr{K} \subset \mathscr{L}$ such that $\mu$ is $\mathscr{K}$-regular and a $\left(\sigma(\widehat{\mathscr{A}})_{\hat{\mu}}, \mathscr{A}\right)$-measurable map $q: \widehat{X} \longrightarrow X$ with $\widehat{K} \subset q^{-1}[K]$ for all $K \in \mathscr{K}$.

Proof. (i) $\Rightarrow$ (ii). Let $\Phi$ be as in (ii), and let $\mathscr{K}$ be a compact lattice such that $\mu$ is $\mathscr{K}$-regular. For all $y \in Y$, put $\mathscr{K}_{y}:=\{K \in \mathscr{K} \mid y \in \Phi(K)\}$. Then $\mathscr{K}_{y} \subset \mathscr{K}$ is nonempty and has f.i.p.; thus, $\bigcap \mathscr{K}_{y} \neq \emptyset$. Select an (arbitrary) element $q(y) \in \bigcap \mathscr{K}_{y}, y \in Y$. This gives us a map $q: Y \longrightarrow X$. We claim that $q$ has the desired properties. To prove this, fix $A \in \mathscr{A}$ and $\epsilon>0$. Since $\mu$ is $\mathscr{K}$-regular, there exist $K \in \mathscr{K}$ and $U \in \mathscr{K}^{c}$ with $K \subset A \subset U$ and 
$\mu(U \backslash K) \leq \epsilon$. An easy consequence of our definition of $q$ is $\Phi(K) \subset q^{-1}[K]$ and, by taking complements, $q^{-1}[U] \subset \Phi(U)$. Thus

$v(\Phi(U) \backslash \Phi(K)) \leq \epsilon$ and $\Phi(K) \subset q^{-1}[K] \subset q^{-1}[A] \subset q^{-1}[U] \subset \Phi(U)$.

This shows $q^{-1}[A] \in \mathscr{B}_{v}$ and, by $\Phi(K) \subset \Phi(A) \subset \Phi(U)$, also $\Phi(A)={ }_{v}$ $q^{-1}[A]$.

(ii) $\Rightarrow$ (iii). If we put $(Y, \mathscr{B}, v):=(\widehat{X}, \sigma(\widehat{\mathscr{A}}), \widehat{\mu})$ and $\Phi:=\widehat{\wedge}: \mathscr{A} \longrightarrow \widehat{\mathscr{A}}$, this implication is obvious.

(iii) $\Rightarrow$ (iv). Take $q$ as in (iii), and put $\mathscr{K}:=\left\{L \in \mathscr{L} \mid \widehat{L} \subset q^{-1}[L]\right\}$. Obviously, $\mathscr{K} \subset \mathscr{L}$ is a lattice satisfying $\widehat{K} \subset q^{-1}[K]$ for all $K \in \mathscr{K}$; since $\widehat{X}$ is compact, $\mathscr{K}$ is also compact. To prove that $\mu$ is $\mathscr{K}$-regular, fix $A \in \mathscr{A}$ and $\epsilon>0$. Then there exists $L_{1} \in \mathscr{L}$ with

(*) $\quad \widehat{L_{1}} \subset \widehat{A} \cap q^{-1}[A] \quad$ and $\quad \widehat{\mu}\left(\widehat{A} \cap q^{-1}[A] \backslash \widehat{L_{1}}\right) \leq \epsilon / 2$.

$\operatorname{Ad}(*)$. Since $\widehat{\mu}$ is $\widehat{\mathscr{A}}_{\delta}$-regular, there exists a decreasing sequence $\left(C_{n}\right)_{n \in \mathbb{N}} \subset \mathscr{A}$ with $\bigcap_{n \in \mathbb{N}} \widehat{C_{n}} \subset \widehat{A} \cap q^{-1}[A]$ and $\widehat{\mu}\left(\widehat{A} \cap q^{-1}[A] \backslash \bigcap_{n \in \mathbb{N}} \widehat{C_{n}}\right) \leq \epsilon / 4$ (N denotes the set of natural numbers without zero). The $\mathscr{L}$-regularity of $\mu$ gives us for $C:=\bigcap_{n \in \mathrm{N}} C_{n} \in \mathscr{A}$ a set $L_{1} \in \mathscr{L}$ with $L_{1} \subset C$ and $\mu\left(C \backslash L_{1}\right)=\widehat{\mu}\left(\widehat{C} \backslash \widehat{L_{1}}\right) \leq$ $\epsilon / 4$. Since $\widehat{L_{1}} \subset \widehat{C} \subset \bigcap_{n \in \mathbb{N}} \widehat{C_{n}}$ and $\widehat{C}=\hat{\mu} \bigcap_{n \in \mathbb{N}} \widehat{C_{n}}$, these inequalities show that $L_{1}$ satisfies $(*)$.

Similarly, there exists $L_{2} \in \mathscr{L}$ with

$$
\widehat{L_{2}} \subset \widehat{L_{1}} \cap q^{-1}\left[L_{1}\right] \quad \text { and } \quad \widehat{\mu}\left(\widehat{L_{1}} \cap q^{-1}\left[L_{1}\right] \backslash \widehat{L_{2}}\right) \leq \epsilon / 4 .
$$

By recursion, we obtain therefore a decreasing sequence $\left(L_{n}\right)_{n \in \mathbb{N}} \subset \mathscr{L}$ with $\widehat{L_{n}} \subset q^{-1}\left[L_{n-1}\right]$ and $\widehat{\mu}\left(\widehat{L_{n-1}} \cap q^{-1}\left[L_{n-1}\right] \backslash \widehat{L_{n}}\right) \leq \epsilon / 2^{n} \quad\left(n \in \mathrm{N}, L_{0}:=A\right)$. Put $K:=\bigcap_{n \in \mathbb{N}} L_{n} \subset A$. Then $K \in \mathscr{L}$ and $\widehat{K} \subset \bigcap_{n \in \mathbb{N}} \widehat{L_{n}} \subset \bigcap_{n \in \mathbb{N}} q^{-1}\left[L_{n-1}\right]$ $=q^{-1}[K]$, i.e., $K \in \mathscr{K}$. Moreover, we obtain

$$
\begin{aligned}
\mu(A \backslash K) & =\mu\left(A \backslash \bigcap_{n \in \mathbb{N}} L_{n}\right)=\mu\left(\bigcup_{n \in \mathbb{N}}\left(L_{n-1} \backslash L_{n}\right)\right) \\
& =\sum_{n \in \mathbb{N}} \mu\left(L_{n-1} \backslash L_{n}\right)=\sum_{n \in \mathbb{N}} \widehat{\mu}\left(\widehat{L_{n}} \backslash \widehat{L_{n}}\right) \\
& \stackrel{(\text { iii) }}{=} \sum_{n \in \mathbb{N}} \widehat{\mu}\left(\widehat{L_{n-1}} \cap q^{-1}\left[L_{n-1}\right] \backslash \widehat{L_{n}}\right) \leq \sum_{n \in \mathbb{N}} \frac{\epsilon}{2^{n}}=\epsilon .
\end{aligned}
$$

This shows (iv).

(iv) $\Rightarrow$ (i). Trivial. 
Remark 1.2. In many aspects Proposition 1.1 is related to [1, Theorem 3.2], a theorem dealing with measures $\mu$ defined on the Baire $\sigma$-field of a completely regular Hausdorff space. The most important ones are:

(a) Since a Baire measure $\mu$ is regular with respect to the $\delta$-lattice of zerosets, a comparison of 1.1 (iv) with [1,3.2(vi)] yields that $\mu$ is compact iff it is H-compact (see [1, p. 559] for the definition).

(b) The defining property of a $\mathrm{H}$-compact Baire measure and condition (ii) of Proposition 1.1 for the abstract measure $\mu$ are similar. Conequently, the same is true for the proofs of 1.1 , (i) $\Rightarrow$ (ii) and [1, Theorem 3.2, (vi) $\Rightarrow$ (i)].

(c) The proof of Proposition 1.1, (iii) $\Rightarrow$ (iv) should be compared with that of [1, Theorem 3.2, (v) $\Rightarrow$ (vi)]. The idea of a recursive construction in the proof of these implications is taken from [10] (Theorem 2.2; cf. [1, Remark (b), p. 564]).

Proposition 1.1 enables us to prove the following Corollary (see [11, Corollary 3] for the corresponding result on semicompact measures).

COROLlary 1.3. Suppose $\mu_{n}, n \in \mathrm{N}$, are measures on a measurable space $(X, \mathscr{A})$ with $\sum_{n \in \mathbb{N}} \mu_{n}(X)<\infty$. Then

$$
\mu_{n} \text { is compact for all } n \in \mathrm{N} \Longleftrightarrow \mu:=\sum_{n \in \mathbb{N}} \mu_{n} \text { is compact. }
$$

Proof. Since " $\Leftarrow$ " is obvious, we only prove " $\Rightarrow$ ". Obviously, each measure $\widehat{\mu_{n}}$ is absolutely continuous with respect to $\widehat{\mu}$; let $h_{n}$ be a corresponding Radon-Nikodym density. We may assume that $\sum_{n \in \mathrm{N}} h_{n}(y)=1$ for all $y \in \widehat{X}$. Since each $\mu_{n}$ is compact, there are lattices $\mathscr{K}_{n}$ and maps $q_{n}, n \in \mathrm{N}$, satisfying the conditions of Proposition 1.1(iv) with $\mathscr{L}:=\mathscr{A}$. First we will show that for all $n \in \mathrm{N}, A \in \mathscr{A}$, and $B \in \sigma(\widehat{\mathscr{A}})$

$$
h_{n} \chi_{q_{n}^{-1}[A]} \quad \text { is } \quad \sigma(\widehat{\mathscr{A}})_{\hat{\mu}} \text {-measurable }
$$

and

$$
\widehat{\mu_{n}}(\widehat{A} \cap B)=\int_{B} h_{n} \chi_{q_{n}^{-1}[A]} d \widehat{\mu} .
$$

Ad (1), (2). Choose, for given $n \in \mathrm{N}$ and $A \in \mathscr{A}$, an increasing sequence $\left(K_{i}\right)_{i \in \mathbb{N}} \subset \mathscr{K}_{n}$ and a decreasing sequence $\left(U_{i}\right)_{i \in \mathbb{N}} \subset \mathscr{K}_{n}^{c}$ with $\bigcup_{i \in \mathbb{N}} K_{i} \subset A \subset$ $\bigcap_{i \in \mathrm{N}} U_{i}$ and $\mu_{n}\left(\bigcup_{i \in \mathrm{N}} K_{i}\right)=\mu_{n}\left(\bigcap_{i \in \mathrm{N}} U_{i}\right)$. Then, for all $B \in \sigma(\mathscr{A})$,

$$
\begin{aligned}
& \widehat{\mu_{n}}(\widehat{A}\cap B)=\sup _{i \in \mathbb{N}} \widehat{\mu_{n}}\left(\widehat{K_{i}} \cap B\right)=\sup _{i \in \mathbb{N}} \int_{\widehat{K_{i}} \cap B} h_{n} d \widehat{\mu}=\int_{B} h_{n} \chi_{\cup_{i \in \mathrm{N}} \widehat{K_{i}}} d \widehat{\mu} \\
& \quad \leq \int_{B} h_{n} \chi_{\bigcap_{i \in \mathrm{N}} \widehat{U}_{i}} d \widehat{\mu}=\inf _{i \in \mathbb{N}} \int_{\widehat{U}_{i} \cap B} h_{n} d \widehat{\mu}=\inf _{i \in \mathbb{N}} \widehat{\mu_{n}}\left(\widehat{U}_{i} \cap B\right)=\widehat{\mu_{n}}(\widehat{A} \cap B) .
\end{aligned}
$$


If we put $B:=\widehat{X}$, this yields $h_{n} \chi_{\bigcup_{i \in \mathrm{N}} \widehat{K_{i}}}=\hat{\mu} h_{n} \chi_{\bigcap_{i \in \mathrm{N}} \widehat{U}_{i}}$. Therefore we obtain (1) and (2) by the inequalities $h_{n} \chi_{\bigcup_{i \in \mathbb{N}} \widehat{K}_{i}} \leq h_{n} \chi_{q_{n}^{-1}[A]} \leq h_{n} \chi_{\bigcap_{i \in \mathbb{N}} \widehat{U}_{i}}$ (note $\widehat{K} \subset q_{n}^{-1}[K]$ for all $\left.K \in \mathscr{K}_{n}\right)$.

Now let $B_{1}:=\left\{h_{1}>0\right\}$ and $B_{k}:=\left\{h_{1}=0, \ldots, h_{k-1}=0, h_{k}>0\right\}$ for $k \geq 2$. The sets $B_{k} \in \sigma(\widehat{\mathscr{A}}), k \in \mathrm{N}$, form a partition of $\widehat{X}$. Therefore, if we put $q(y):=q_{k}(y)$ for $y \in B_{k}, k \in \mathrm{N}$, we obtain a map $q: \widehat{X} \longrightarrow X$. We will show that $q$ satisfies (iii) of Proposition 1.1. Fix $A \in \mathscr{A}$. Then $q^{-1}[A]=$ $\bigcup_{k \in \mathbb{N}}\left(B_{k} \cap q^{-1}[A]\right)=\bigcup_{k \in \mathbb{N}}\left(B_{k} \cap q_{k}^{-1}[A]\right)$. Since each $h_{k} \chi_{q_{k}^{-1}[A]}$ is $\sigma\left(\widehat{A}_{)^{-}}\right.$ measurable (recall (1)), $B_{k} \cap q_{k}^{-1}[A] \in \sigma(\widehat{\mathscr{A}})_{\hat{\mu}}$. Thus, $q^{-1}[A] \in \sigma(\widehat{\mathscr{A}})_{\hat{\mu}}$; i.e., $q$ is $\left(\sigma(\widehat{\mathscr{A}})_{\hat{\mu}}, \mathscr{A}\right)$-measurable. Given $k \in \mathrm{N}$, we obtain by the equation $\widehat{\mu}=\sum_{n \in \mathbb{N}} \widehat{\mu_{n}}$ and by (2) (put there $B:=\widehat{A} \cap B_{k}$ )

$$
\begin{aligned}
\widehat{\mu}\left(\widehat{A} \cap B_{k}\right)=\sum_{n \in \mathbb{N}} \widehat{\mu_{n}}\left(\widehat{A} \cap B_{k}\right) & =\sum_{n \in \mathbb{N}} \int_{\widehat{A} \cap B_{k}} h_{n} \chi_{q_{n}^{-1}[A]} d \widehat{\mu} \\
& =\int_{\widehat{A} \cap B_{k}} \sum_{n \in \mathbb{N}} h_{n} \chi_{q_{n}^{-1}[A]} d \widehat{\mu} .
\end{aligned}
$$

This shows that $\sum_{n \in \mathbb{N}}\left(h_{n} \chi_{q_{n}^{-1}[A]}\right)(y)=1$ holds for $\widehat{\mu}$-almost every $y \in \widehat{A} \cap B_{k}$. Let $y_{0} \in \widehat{A} \cap B_{k}$ with $\sum_{n \in \mathbb{N}}\left(h_{n} \chi_{q_{n}^{-1}[A]}\right)\left(y_{0}\right)=1$ be given. Since $h_{k}\left(y_{0}\right)>0$ and $\sum_{n \in \mathbb{N}} h_{n}\left(y_{0}\right)=1$, we have $y_{0} \in q_{k}^{-1}[A]$. Thus, $\widehat{A} \cap B_{k} \subset_{\hat{\mu}} q_{k}^{-1}[A] \cap B_{k}=$ $q^{-1}[A] \cap B_{k} \subset q^{-1}[A]$, and hence $\widehat{A} \subset_{\hat{\mu}} q^{-1}[A]$.

We notice some further properties of compact measures, mainly obtained by Corollary 1.3.

REMARK 1.4. Let $\mu, v$ be measures. If $v$ is absolutely continuous with respect to $\mu$ and $\mu$ is compact, then $v$ is compact, too. Since $\mu \vee v, \mu \wedge v \leq \mu+v$, this shows together with Corollary 1.3 that the supremum and the infimum of two compact measures is compact again. Obviously, the same is true for the direct product of two compact measures. Moreover, using Corollary 1.3 again, we can prove (completely analogous to [11, Corollary 4]) that for every countable family $M$ of compact measures there is always a compact lattice $\mathscr{K}$ such that each $\mu \in M$ is $\mathscr{K}$-regular.

\section{The relation between compact measures and measurable weak sections and its consequences}

In this section, we prove our main results on compact measures. Let us begin with the following central definition: Suppose $(X, \mathscr{A})$ is a measurable space, $(Y, \mathscr{B}, v)$ is a measure space, and $p: X \longrightarrow Y$ is $(\mathscr{A}, \mathscr{B})$-measurable; then, following [3] and [4], we call a map $q: Y \longrightarrow X$ a measurable weak section 
for $p$ provided $q$ is $\left(\mathscr{B}_{v}, \mathscr{A}\right)$-measurable and $q^{-1}\left[p^{-1}[B]\right]={ }_{v} B$ holds for all $B \in \mathscr{B}$.

To prove our theorems, we need a well-known criterion due to D. Plachky: A measure $\mu \in M(p, v)$ (the convex set of all preimage measures of $v$ ) is an extreme point, i.e. a member of ex $M(p, v)$, iff for all $A \in \mathscr{A}$ there exists $B \in \mathscr{B}$ with $A={ }_{\mu} p^{-1}[B]$ (see [12] or, e.g., [5, Lemma 3]). In the sequal, we will simply write " $(\mathrm{Pl})$ " if we refer to this fact.

To become more familiar with the above definition and with $(\mathrm{Pl})$, let us discuss a simple, but instructive

Example 2.1. Let $(X, \mathscr{A}):=\left([0,1],\left\{A \subset X \mid A\right.\right.$ or $A^{c}$ is countable $\left.\}\right)$ and $\mu(A):=0$ if $A$ is countable and $\mu(A):=1$ for all other sets $A \in$ $\mathscr{A}$. Furthermore, let us put $(Y, \mathscr{B}):=(X,\{\emptyset, X\}), v(\emptyset):=0, v(Y):=1$, and $p:=\mathrm{id}_{[0,1]}$. By (Pl), we obtain $\mu \in \operatorname{ex} M(p, v)$; but, since every weak measurable section $q$ of $p$ is constant, we cannot obtain $\mu$ in the way $q(v)$, which yields a Dirac measure. However, if we note that a 2-valued nontrivial measure is compact iff it is a Dirac measure ([13, Theorem 4.6]), we can see that exactly the compact measures $\mu \in \operatorname{ex} M(p, v)$ are of the form $q(v)$. (The content of Corollary 2.9 is that this fact remains true even in a general situation.)

Our first result generalizes [4, Theorem 3].

THEOREM 2.2. Let $(X, \mathscr{A}, \mu)$ be a compact measure space, $(Y, \mathscr{B}, v)$ be a measure space, and $p: X \longrightarrow Y$ be an $(\mathscr{A}, \mathscr{B})$-measurable map with $p(\mu)=v$. Then the following statements are equivalent:

(i) $\mu \in \operatorname{ex} M(p, v)$.

(ii) There exists a measurable weak section $q: Y \longrightarrow X$ for $p$ with $q(v)=\mu$.

Proof. (i) $\Rightarrow$ (ii). We may assume that the measure $v$ is nontrivial. Since $\mu$ is extremal, for every $A \in \mathscr{A}$ there exists, by $(\mathrm{Pl})$, a set $\Psi(A) \in \mathscr{B}$ with $A={ }_{\mu} p^{-1}[\Psi(A)]$. This gives us a map $\Psi: \mathscr{A} \longrightarrow \mathscr{B}$ with the following properties:

- $\Psi(\emptyset)={ }_{v} \emptyset, \Psi(X)={ }_{v} Y$;

- $\Psi\left(A_{1} \cap A_{2}\right)={ }_{v} \Psi\left(A_{1}\right) \cap \Psi\left(A_{2}\right)$ for all $A_{1}, A_{2} \in \mathscr{A}$;

- $\Psi\left(A^{c}\right)={ }_{v}(\Psi(A))^{c}$ for all $A \in \mathscr{A}$.

Let $l$ be a lifting for $\mathscr{B}_{v}$ (see, e.g., [6, Chapter IV]); then $\Phi:=l \circ \Psi$ : $\mathscr{A} \longrightarrow \mathscr{B}_{v}$ is an algebra homomorphism with $(*) v(\Phi(A))=v(\Psi(A))=$ $\mu\left(p^{-1}[\Psi(A)]\right)=\mu(A)$ for all $A \in \mathscr{A}$. Therefore, according to Proposition 1.1, (i) $\Rightarrow$ (ii), there exists a $\left(\mathscr{B}_{v}, \mathscr{A}\right)$-measurable map $q: Y \longrightarrow X$ with $q^{-1}[A]={ }_{\nu} \Phi(A)$ for all $A \in \mathscr{A}$. Together with (*) this gives us $q(\nu)=\mu$. 
By definition of $\Psi$, we have $p^{-1}[B]={ }_{\mu} p^{-1}\left[\Psi\left(p^{-1}[B]\right)\right]$ for all $B \in \mathscr{B}$, and therefore $B={ }_{\nu} \Psi\left(p^{-1}[B]\right)={ }_{\nu} \Phi\left(p^{-1}[B]\right)={ }_{\nu} q^{-1}\left[p^{-1}[B]\right]$. Hence $q$ is a measurable weak section for $p$.

(ii) $\Rightarrow$ (i) (this implication is known; see, e.g., [4, Remark, p. 74]). Since $v(B)=v\left(q^{-1}\left[p^{-1}[B]\right]\right)=\mu\left(p^{-1}[B]\right)$ for all $B \in \mathscr{B}$, we have $\mu \in M(p, v)$. Moreover, for every $A \in \mathscr{A}$ there exists a set $B \in \mathscr{B}$ with $q^{-1}[A]={ }_{\nu} B={ }_{v}$ $q^{-1}\left[p^{-1}[B]\right]$. Thus, $A={ }_{\mu} p^{-1}[B]$. By $(\mathrm{Pl})$, this gives us $\mu \in \operatorname{ex~} M(p, v)$.

For the proof of a converse of Theorem 2.2 (and for Theorem 2.6) we need the following Lemma. There, given a measurable space $(X, \mathscr{A})$ and $x \in X$, we write $p_{\mathscr{A}}(x)$ for the unique element contained in the nonempty set $\bigcap\{\widehat{A} \mid$ $A \in \mathscr{A}, x \in A\}$.

Lemma 2.3. Let $(X, \mathscr{A}, \mu)$ be a measure space, and let $p_{\mathscr{A}}: X \ni x \mapsto$ $p_{\mathscr{A}}(x) \in \widehat{X}$. Then $p_{\mathscr{A}}$ is an $(\mathscr{A}, \sigma(\widehat{\mathscr{A}}))$-measurable map with $p_{\mathscr{A}}^{-1}[\widehat{A}]=A$ for all $A \in \mathscr{A}$. Moreover, $\mu \in \operatorname{ex} M\left(p_{\mathscr{A}}, \widehat{\mu}\right)$ holds.

Proof. Let $A \in \mathscr{A}$. Since $p_{\mathscr{A}}(x) \in \widehat{A}$ iff $x \in A$, we have $p_{\mathscr{A}}^{-1}[\widehat{A}]=A$. This shows that $p_{A}$ is $(\mathscr{A}, \sigma(\widehat{\mathscr{A}})$ )-measurable and, moreover, that the set $M\left(p_{\mathscr{A}}, \widehat{\mu}\right)$ is a singleton containing $\mu$; hence $\mu \in \operatorname{ex} M\left(p_{\mathscr{A}}, \widehat{\mu}\right)$.

Theorem 2.4. Let $(X, \mathscr{A}, \mu)$ be a measure space. Suppose that for every measure space $(Y, \mathscr{B}, v)$ and every $(\mathscr{A}, \mathscr{B})$-measurable map $p: X \longrightarrow Y$ satisfying $\mu \in \operatorname{ex} M(p, v)$ there exists a measurable weak section $q: Y \longrightarrow X$ for $p$ with $q(v)=\mu$. Then $\mu$ is compact.

Proof. Put $(Y, \mathscr{B}, v):=(\widehat{X}, \sigma(\widehat{\mathscr{A}}), \widehat{\mu})$ and $p:=p_{\mathscr{A}}: X \longrightarrow Y$. By Lemma $2.3, p$ is an $(\mathscr{A}, \mathscr{B})$-measurable map with $\mu \in \operatorname{ex~} M(p, v)$. According to our assumption, there exists therefore a $\left(\sigma(\widehat{\mathscr{A}})_{\hat{\mu}}, \mathscr{A}\right)$-measurable map $q$ : $\widehat{X} \longrightarrow X$ with $\widehat{A}=\hat{\mu} q^{-1}\left[p_{\mathscr{A}}^{-1}[\widehat{A}]\right]=q^{-1}[A]$ for all $A \in \mathscr{A}$. This shows that $\mu$ is compact (Proposition 1.1, (iii) $\Rightarrow$ (i)).

By [11, Theorem 2], the image $p(\lambda)$ of a semicompact measure $\lambda$ is semicompact again. The following simple example shows that this is no longer true for compact measures.

EXAMPLE 2.5. Let $\lambda$ be the Lebesgue measure on the usual Borel $\sigma$-algebra of $[0,1]$, and let $(X, \mathscr{A}, \mu)$ and $p$ be as in Example 2.1. Then $p(\lambda)=\mu$, but $\mu$ is not compact.

Of course, in this example $\lambda$ is not an extremal preimage measure. The next result shows that this fact is crucial.

Theorem 2.6. Let $(X, \mathscr{A}, \mu)$ be a compact measure space, $(Y, \mathscr{B}, v)$ be a measure space, and $p: X \longrightarrow Y$ be an $(\mathscr{A}, \mathscr{B})$-measurable map such that $\mu \in \operatorname{ex} M(p, v)$. Then $p(\mu)=v$ is compact. 
Proof. Put $\tilde{p}:=p_{\mathscr{B}} \circ p: X \longrightarrow \widehat{Y}$ (recall Lemma 2.3). Then $\mu \in$ ex $M(\tilde{p}, \widehat{v})$. According to Theorem 2.2, (i) $\Rightarrow$ (ii), there exists a $\left(\sigma(\widehat{\mathscr{B}})_{\hat{v}}, \mathscr{A}\right)$ measurable map $\tilde{q}: \widehat{Y} \longrightarrow X$ such that $\widehat{B}=\hat{v} \tilde{q}^{-1}\left[\tilde{p}^{-1}[\widehat{B}]\right]$ holds for all $B \in \mathscr{B}$. Now, put $q:=p \circ \tilde{q}$; then $q: \widehat{Y} \longrightarrow Y$ is $\left(\sigma(\widehat{B})_{\hat{v}}, \mathscr{B}\right)$-measurable with $q^{-1}[B]=\tilde{q}^{-1}\left[p^{-1}[B]\right]=\tilde{q}^{-1}\left[p^{-1}\left[p_{\mathscr{B}}^{-1}[\widehat{B}]\right]\right]=\tilde{q}^{-1}\left[\tilde{p}^{-1}[\widehat{B}]\right]=\hat{v} \widehat{B}$ for all $B \in \mathscr{B}$. Again, Proposition 1.1 proves the theorem.

An immediate consequence of Theorem 2.6 and $(\mathrm{Pl})$ is

Remark 2.7. Suppose $(X, \mathscr{A}, \mu)$ is a compact measure space and $\mathscr{B} \subset \mathscr{A}$ is a $\sigma$-algebra such that for all $A \in \mathscr{A}$ there exists $B \in \mathscr{B}$ with $B={ }_{\mu} A$; then $\mu \mid \mathscr{B}$ is compact. E.g., if the completion of a measure $\mu$ is compact, then $\mu$ is compact, too. (The last fact follows already from Proposition 1.1, (i) $\Rightarrow$ (iv).)

In the case of a completely regular Hausdorff space the next result is Proposition 3.1 of [1] (cf. Remark 1.2(a)). Recall the following definition (see, e.g., [2]): A Baire measure $v$, i.e. a finite measure defined on the $\sigma$-algebra $\Im(X)$ generated by the lattice $3(X)$ of zero-sets in an (arbitrary) topological space $X$, is called tight provided for every $\epsilon>0$ there exists a compact set $K \subset X$ such that $v^{*}(K) \geq v(X)-\epsilon$ (as usual, $v^{*}$ denotes the outer measure associated with $v$ ).

Corollary 2.8. Every tight Baire measure on a topological space $X$ is compact with respect to a sublattice of $3(X)$.

Proof. Let $\nu \mid \mathfrak{C}(X)$ be tight, and let $\left(K_{n}\right)_{n \in \mathbb{N}}$ be an increasing sequence of compact sets with $\sup _{n \in \mathbb{N}} v^{*}\left(K_{n}\right)=v(X)$. Since $v$ is $8(X)$-regular, we only have to prove that $v$ is compact (Proposition 1.1, (i) $\Rightarrow$ (iv)). Put $\mathscr{A}:=$ $\sigma\left(\widetilde{S}(X) \cup\left\{K_{n} \mid n \in \mathrm{N}\right\}\right)$. According to [8, Theorem 2], there exists a (unique) measure $\mu$ on $\mathscr{A}$ with $\mu\left(K_{n}\right)=v^{*}\left(K_{n}\right)$ for all $n \in \mathrm{N}$ and $\mu \mid \subseteq(X)=v$. Then $\mu$ is an extremal extension of $v$ (see, e.g., [8, Remark 2]). By Theorem 2.6, it remains to show that $\mu$ is compact. We put $\mathscr{K}_{n}:=\left\{K_{n} \cap Z \mid Z \in B(X)\right\}, n \in \mathrm{N}$, and $\mathscr{K}^{\prime}:=\bigcup_{n \in \mathrm{N}} \mathscr{K}_{n}$. Since $K_{n}$ is compact and $K_{n} \subset K_{n+1}, n \in \mathrm{N}$, the family $\mathscr{K}^{\prime}$ is compact. Let $\mathscr{K}$ be the $\delta$-lattice generated by $\mathscr{K}^{\prime}$. Then $\mathscr{K} \subset \mathscr{A}$ is compact, too (see, e.g., [14, Corollary 3.2]). We will show that $\mu$ is $\mathscr{K}$-regular. Put $\mathscr{A}_{0}:=\left\{A \in \mathscr{A} \mid A\right.$ and $A^{c}$ satisfy $\left.(*)\right\}$, where (*) holds for $A \in \mathscr{A}$ iff $\mu(A)=\sup \{\mu(K) \mid K \in \mathscr{K}, K \subset A\}$. Using well-known arguments, we see that $\mathscr{A}_{0}$ is a $\sigma$-algebra. If we can show that $\subseteq(X) \cup\left\{K_{n} \mid n \in \mathrm{N}\right\} \subset \mathscr{A}_{0}$, the proof is complete.

Fix $B \in \mathfrak{S}(X)$ and $\epsilon>0$. Since every Baire measure in a space $X$ is $8(X)$ regular, there exists $Z \in B(X)$ with $Z \subset B$ and $\mu(B \backslash Z)=v(B \backslash Z) \leq \epsilon / 2$. Furthermore, there exists $N \in \mathrm{N}$ with $\mu\left(K_{N}^{c}\right) \leq \epsilon / 2$. For $K:=Z \cap K_{N} \in \mathscr{K}$ we obtain therefore $K \subset B$ and $\mu(B \backslash K) \leq \epsilon$. Hence $B$ satisfies $(*)$; for the 
same reasons, this is also true for $B^{c}$. Thus, $B \in \mathscr{A}_{0}$. Finally, let $K_{n}, n \in \mathrm{N}$, and $\epsilon>0$ be given. As $K_{n} \in \mathscr{K}$, we only have to consider $K_{n}^{c}$. Since $\mu\left(K_{n}\right)=$ $\nu^{*}\left(K_{n}\right)$, there exists $U \in 3(X)^{c}$ with $U \supset K_{n}$ and $\mu\left(U \backslash K_{n}\right) \leq \epsilon / 2$ (recall that $\mu \mid \mathfrak{C}(X)=v$ and that $v$ is $3(X)$-regular). Again, choose $N \in \mathrm{N}$ with $\mu\left(K_{N}^{c}\right) \leq \epsilon / 2$, and put $K:=K_{N} \cap U^{c} \in \mathscr{K}$. Then we have $K \subset U^{c} \subset K_{n}^{c}$ and $\mu\left(K_{n}^{c} \backslash K\right)=\mu\left(K_{n}^{c} \backslash U^{c}\right)+\mu\left(U^{c} \backslash\left(K_{N} \cap U^{c}\right)\right) \leq \epsilon / 2+\epsilon / 2=\epsilon$. This shows that $K_{n} \in \mathscr{A}_{0}$.

A motivation for our last result is provided by Example 2.1: There we recognized that the set $\{q(v) \mid q$ is a weak measurable section for $p\}$ and the set of all extremal compact preimage measures of $v$, denoted by ex $M_{c}(p, v)$ note that, according to Corollary 1.3, the set $M_{c}(p, v)$ of all compact preimage measures is convex and a face in $M(p, v)$ - coincide. Of course, this is not always the case: consider, e.g., a noncompact measure $v$ on a $\sigma$-algebra $\mathscr{B}$, and put $\mu:=v, \mathscr{A}:=\mathscr{B}$, and $p=$ id. But, in Example 2.1, $v$ is compact; and this is crucial:

Corollary 2.9. Let $(X, \mathscr{A})$ be a measurable space, $(Y, \mathscr{B}, v)$ be a compact measure space, and $p: X \longrightarrow Y$ be an $(\mathscr{A}, \mathscr{B})$-measurable map. Then

$$
\text { ex } M_{c}(p, v)=\{q(v) \mid q \text { is a weak measurable section for } p\} \text {. }
$$

Proof. By Theorem 2.2, (i) $\Rightarrow$ (ii), the inclusion " $\subset$ " is obvious. Let now $q$ be a weak measurable section for $p$. By Theorem 2.2, (ii) $\Rightarrow$ (i), $q(v) \in \operatorname{ex} M(p, v)$ holds. We claim that $q(v)$ is also compact. Since $v$ is compact, this is true if $v \in \operatorname{ex~} M(q, q(v))$ (recall Theorem 2.6). The relation $v \in M(q, q(v))$ is obvious. Therefore, by (Pl), we have to prove that for all $B \in \mathscr{B}_{v}$ there exists a set $A \in \mathscr{A}$ with $B={ }_{\nu} q^{-1}[A]$. Obviously, we may assume that $B \in \mathscr{B}$; but then we can put $A:=p^{-1}[B] \in \mathscr{A}$, and obtain the desired equation $B={ }_{\nu} q^{-1}\left[p^{-1}[B]\right]=q^{-1}[A]$.

\section{REFERENCES}

1. Babiker, A. G. A. G. and Graf, S., Homomorphism-compact spaces, Canad. J. Math. 35 (1983), no. 3, 558-576.

2. Edgar, G. A., Disintegration of measures and the vector-valued Radon-Nikodym theorem, Duke Math. J. 42 (1975), 447-450.

3. Edgar, G. A., Measurable weak sections, Illinois J. Math. 20 (1976), 630-646.

4. Graf, S., Induced $\sigma$-homomorphisms and a parametrisation of measurable sections via extremal preimage measures, Math. Ann. 247 (1980), 67-80.

5. Hackenbroch, W., Dilated sections and extremal preimage measures, Arch. Math. 43 (1984), 434-439.

6. Ionescu-Tulcea, A. and C., Topics in the Theory of Lifting, Springer, Berlin-Heidelberg-New York, 1968. 
7. Koumoullis, G. and Prikry, K., The Ramsey property and measurable selections, J. London Math. Soc. 28 (1983), 203-210.

8. Lipecki, Z., On unique extensions of positive additive set functions, Arch. Math. 41 (1983), 71-79.

9. Marczewski, E., On compact measures, Fund. Math. 40 (1953), 113-124.

10. Pachl, J., Disintegration and compact measures, Math. Scand. 43 (1978), 157-168.

11. Pachl, J., Two classes of measures, Colloq. Math. 42 (1979), 331-340.

12. Plachky, D., Extremal and monogenic additive set functions, Proc. Amer. Math. Soc. 54 (1976), 193-196.

13. Render, H., Pushing down Loeb measures, Math. Scand. 72 (1993), 61-84.

14. Ross, D., Compact measures have Loeb preimages, Proc. Amer. Math. Soc. 115 (1992), 365-370.

15. Schwartz, L., Radon Measures on Arbitrary Topological Spaces and Cylindrical Measures, Oxford University Press, London, 1973.

MATHEMATISCHES INSTITUT

DER UNIVERSITÄT MÜNCHEN

THERESIENSTR. 39

D-80333 MÜNCHEN

GERMANY

E-mail: rinkewit@rz.mathematik.uni-muenchen.de 